\title{
A Surface Resistance Effect on the Fabrication of Dye-sensitized Solar Cell with Various Widths*
}

\author{
Jin-Young CHOI*1, Hyun-Woong Seo*1, Mi-Jeong KIM*1, \\ Ji-Young SIM*1, Dong-Yoon LEE*2 and Hee-Je KIM*1 \\ ${ }^{* 1}$ Department of Electrical Engineering, Pusan National University Jangjeon-dong, \\ Geumjeong-gu, Busan 609-735, Republic of Korea \\ *2Advanced Materials \& Application research Laboratory, Korea Electrotechnology Research Institute, \\ 28-1, Seongju-dong, Changwon-city, Kyongnam, 641-120, Republic of Korea \\ (Received July 15, 2006, Accepted February 10, 2007)
}

Sputter deposition followed by surface treatment was studied using reactive RF plasma as a method for preparing titanium oxide $\left(\mathrm{TiO}_{2}\right.$ ) films on the FTO (fluorine doped tin oxide, $\mathrm{SnO}_{2}: \mathrm{F}$ ) substrate for dye-sensitized solar cells (DSCs). Anatase structure $\mathrm{TiO}_{2}$ films deposited by reactive $\mathrm{RF}$ magnetron sputtering under the conditions of $\mathrm{Ar} / \mathrm{O}_{2}(5 \%)$ mixing gas, $\mathrm{RF}$ power of $600 \mathrm{~W}$ and substrate temperature of $400^{\circ} \mathrm{C}$ were surface-treated by inductive coupled plasma (ICP) with Ar/ $\mathrm{O}_{2}$ mixing gas at substrate temperature of $400^{\circ} \mathrm{C}$, and thus the films were applied to the DSCs. We have chosen a solar cell width as a variable of a large-scaled DSCs and confirmed electric characteristics of an individual cell. As a result, the higher the internal resistance of DSC becomes, the wider the width gets. Internal resistance makes it difficult to collect photoelectron generated from dye. Ultimately up sizing DSC causes the increase of internal resistance and then has a bad effect on the cell characteristics.

\section{Introduction}

Nanocrystalline dye-senstitized solar cells (DSCs) are promising candidates for low-cost photovoltaic devices $^{1)}$. Several prototypes have been presented, but no large-scale production has yet appeared as a consequence of the many problems that these devices are still facing. It is claimed that the unique properties of the nanocrystalline DSCs stem from the ability to achieve an interaction of the dye and the electrolyte with all interconnected nanoparticles.

DSCs are based on a wide band-gap semiconductor material with a very large internal surface to which dye molecules are attached. Titanium oxide $\left(\mathrm{TiO}_{2}\right)$ is an important material in the construction of DSCs because of its large surface area, sufficient light absorption and high photoelectrical response as a porous photo-electrode material of $\mathrm{DSCs}^{2}$. The electron is excited from the valence band to the conduction band, when $\mathrm{TiO}_{2}$ is irradiated with ultraviolet radiation of about $380 \mathrm{~nm}$ or less wavelength, and electron-hole pairs are created.

DSCs typically consist of a dye sensitized $\mathrm{TiO}_{2}$ electrode in contact with an electrolyte and completed by an inert counter electrode. Remarkable progress regarding the efficiency of DSCs was made possible by employing porous nanocrystalline $\mathrm{TiO}_{2}$ prepared by sol-gel methods. The porous network of the $\mathrm{TiO}_{2}$ nanoparticles prepared by this technology result in extremely large surface area, greatly enhancing absorption of light as compared to a monolayer of dye adsorbed on a flat unstructured surface of the same size. However, the randomly porous structure of $\mathrm{TiO}_{2}$ electrode gives rise to several

* Presented at ISDEIV 2006, Matsue, Japan, September 25-29, 2006 undesired characteristics, such as low conductivity and charge density. Recently, DC magnetron sputtering technique has been used to deposit porous $\mathrm{TiO}_{2}$ thin films with large surface area for use in DSCs. The sputtered films yielded a high photocurrent when incorporated into DSCs configuration, although energy conversion efficiency was not still high ${ }^{3}$.

Commercial solar cells like poly-crystalline Si solar cells have a current-collecting grid that was made by silver solder or conductive printing paste to reduce their high surface resistance. In the case of dye-sensitized solar cells, there are some precedents with the metal grid protected by resin or glass-ceramic to be not attacked from redox electrolyte ${ }^{4-5)}$.

In this study, different square sizes of DSCs with various widths were especially investigated. The surface resistance, Isc, Voc and fill factor (FF) of DSCs were measured and discussed in association with the different widths.

\section{Experimental procedure}

\subsection{Preparation of $\mathrm{TiO}_{2}$ films}

The process was roughly divided into two in this experiment. The primary process formed $\mathrm{TiO}_{2}$ films of anatase crystal structure by the RF reactive magnetron sputter system as shown in Fig. 1. The secondary process was the plasma surface treatment using immersed type single-turn ICP. The RF magnetron sputter system can be briefly described as follows. The chamber was of stainless steel of internal diameter $300 \mathrm{~mm}$. The RF power $\left(P_{\mathrm{rf}}\right)$ of $600 \mathrm{~W}$ was supplied to a $\mathrm{TiO}_{2}$ target having diameter of $100 \mathrm{~mm}$. The distance between the target and substrate was $150 \mathrm{~mm}$.

A temperature controller and heater controlled the substrate temperature. Cooling water is circulated 
through the target and chamber to prevent overheating during deposition. At first, the chamber was exhausted vacuum to $2 \times 10^{-6}$ Torr or less. The temperature of the $20 \mathrm{~mm}$ (20 mm FTO (fluorine doped tin oxide, $\mathrm{SnO}_{2}: \mathrm{F}$ ) substrate was maintained at $400^{\circ} \mathrm{C}$. According to the Dalton's partial pressure law, we used the mixing gas of $100 \mathrm{~kg} / \mathrm{cm}^{2}$ : Ar $95 \mathrm{~kg} / \mathrm{cm}^{2}, \mathrm{O}_{2} 5 \mathrm{~kg} / \mathrm{cm}^{2}$. The $\mathrm{Ar} / \mathrm{O}_{2}(5$ $\%)$ with $5: 1$ pressure ratio gas was introduced to 18 mTorr, and the $P_{\mathrm{rf}}$ of $600 \mathrm{~W}$ was applied to target. And hence, the deposited $\mathrm{TiO}_{2}$ films were taken out of the chamber to confirm the structure and thickness of the films.

\subsection{The assembly of the dye-sensitized solar cell}

Many studies have been reported on modifying the surface properties of $\mathrm{TiO}_{2}$ by various methods, including chemical processes (aquageria, degreasing, and RCA protocol) and physical treatments using oxygen or argon plasma ${ }^{6}$. Among them, $\mathrm{Ar} / \mathrm{O}_{2}$ plasma was considered as a promising treatment because it results in the highest work function, the lowest sheet resistance and the smoothest surface ${ }^{7}$. Consecutively, a procedure of the plasma surface treatment is indicated as second process. The prepared $\mathrm{TiO}_{2}$ films were dye-sensitized with a dye solution. Before immersing into the dye solution, the films were plasma-treated to remove physisorbed impurities and promote surface activity. The $\mathrm{TiO}_{2}$ films are reset in an ICP chamber, which was described in detailed in our previous report ${ }^{8)}$, and the $\mathrm{Ar} / \mathrm{O}_{2}$ mixing gas were introduced to a total pressure of $18 \mathrm{~m}$ Torr and $P_{\mathrm{rf}}$ of 400

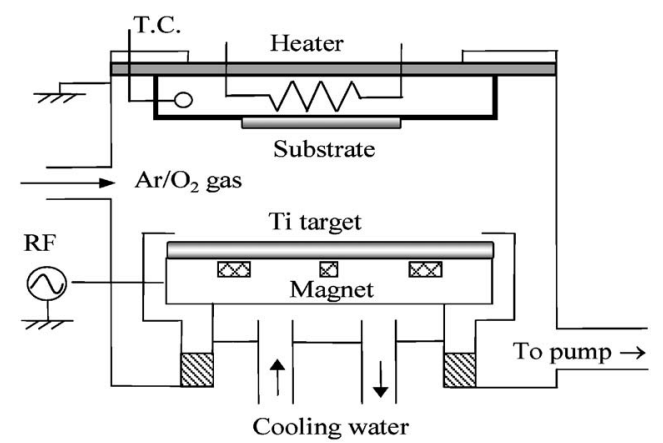

Fig. 1 Schematic diagram of RF magnetron sputter system.
W was applied, and plasma surface treatment was performed for $1 \mathrm{~h}$. The temperature of the substrate was made to be $400^{\circ} \mathrm{C}$ After the plasma treatment, the resulting $\mathrm{TiO}_{2}$ properties were investigated by X-ray diffractometry (XRD), Scanning electron microscopy (SEM).

In this study, we designed DSCs with an active area of same height and the different width. To confirm the influence of electronic flow by surface resistance, we chose the width of a cell as the variable.

That is, the width of DSC was changed as a variable for large-sized solar cell. The height of an active area for all DSCs is $60 \mathrm{~mm}$ and the width makes different to 1, 3, $5,7,10,20 \mathrm{~mm}$ respectively. And we expected effect that increases in surface resistance. Figure 2 represents an appearance and an active area of DSCs used in this study.

FTO glass with approximately $9.3 \Omega / \mathrm{cm}^{2}$ of sheet resistance is cleaned and prepared in advance. $\mathrm{TiO}_{2}$ paste is coated by screen printing method on it. And then it is sintered for 30 minutes at $450^{\circ} \mathrm{C}$. The dye of cis-bis (isothiocyanato) bis (2,2' -bipyridyl-4,4' -dicarboxylato)ruthenium(II)(N3) dissolved on ethanol is adsorbed on the nano-porous $\mathrm{TiO}_{2}$ electrode film manufactured through the previous process. The Pt catalyst is coated by RF sputtering method. The working and counter electrode are sealed by the adhesive (SolaronixSA, Amoil 4) in a sandwich. The gap between two electrodes is filled with the electrolyte (Iodolyte TG-50). And then DSCs for experiment is completed after sealing the hole to fill up the electrolyte. Figure 3 shows structure of and DSCs for experiment. Electrical properties of DSCs for experiment were measured by Keithlley 2420 source meter on Solar Simulator $\left(100 \mathrm{~mW} / \mathrm{cm}^{2}\right)$.

\section{Result and discussion}

Structural characterization of the plasma-treated $\mathrm{TiO}_{2}$ films was performed by XRD and the result was shown in Fig. 4. The result before plasma treatment showed a prominent peak assigned to anatase (101), as well as two peaks due to the rutile (110) and (101) reflections. The film after plasma treatment displayed an expectedly weak anatase peak.

Surface morphologies were studied with SEM. Figure 5(a) and (b) show the SEM images on the surface and

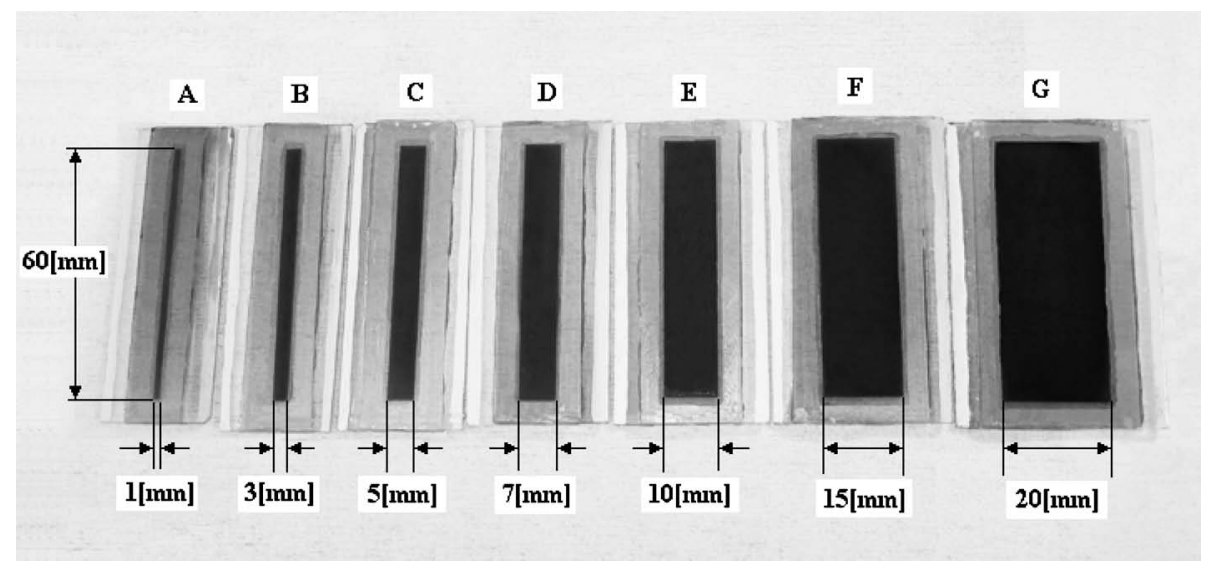

Fig. 2 An appearance of DSCs for measurements. 


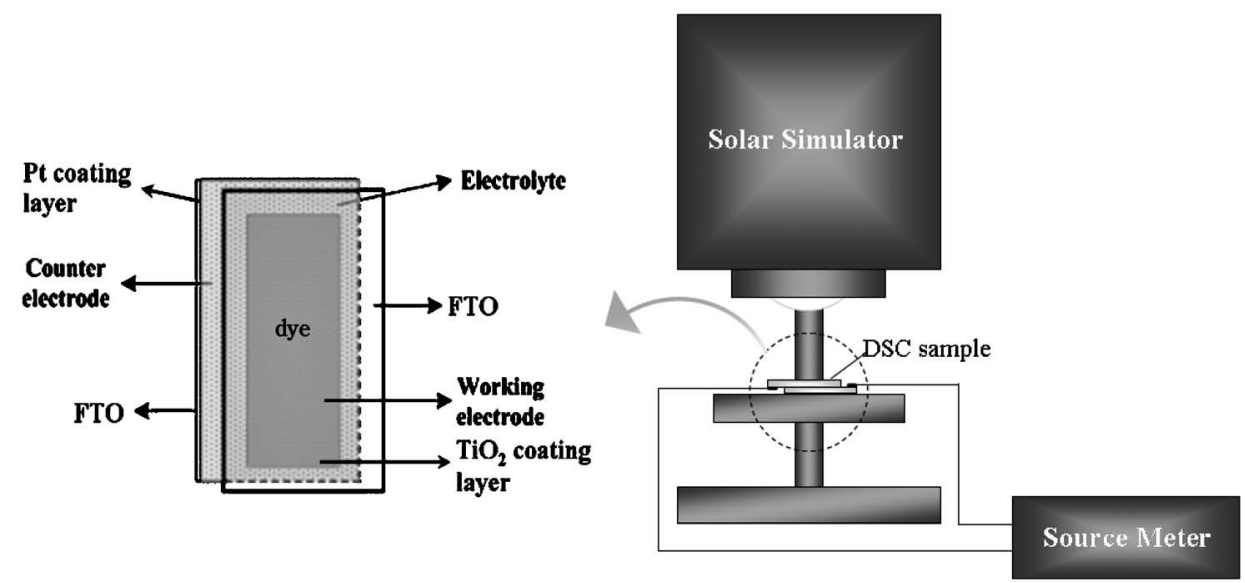

Fig. 3 Structure and of DSC for measurements.

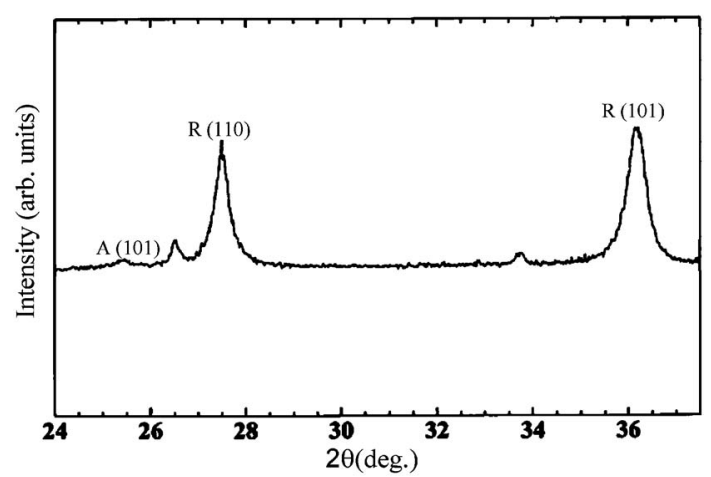

Fig. 4 XRD spectra for plasma-treated $\mathrm{TiO}_{2}$ film. A: Anatase, R: Rutile

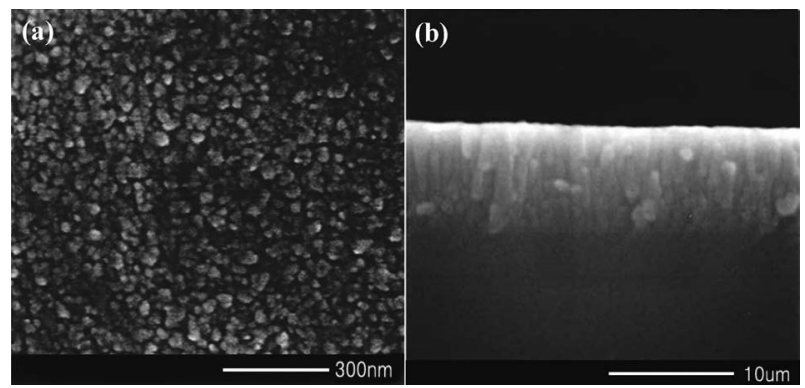

Fig. 5 SEM images of the untreated $\mathrm{TiO}_{2}$ film. (a) Surface, (b) Cross section.

cross section of the prepared $\mathrm{TiO}_{2}$ film, respectively. The micrograph gives clear evidence for the growth of $\mathrm{TiO}_{2}$ crystal. As seen from Fig. 5(a), rough surfaces with wellrounded particle were apparent, and the particle size of about $25 \mathrm{~nm}$ could be confirmed. In Fig. 5(b), the micrograph in the cross section shows that the crystals developed in a pillar shape at the top of the film. Figure 6 shows the surface SEM image after plasma treatment. The crystal structure shows much clearer. Also, it demonstrates that the distances between the gains were lengthened by the partial removal of the surface contaminated layer. This contributes to the greater contact area with the water and air.

Figure 7 shows TEM image of the $\mathrm{TiO}_{2}$ film prepared

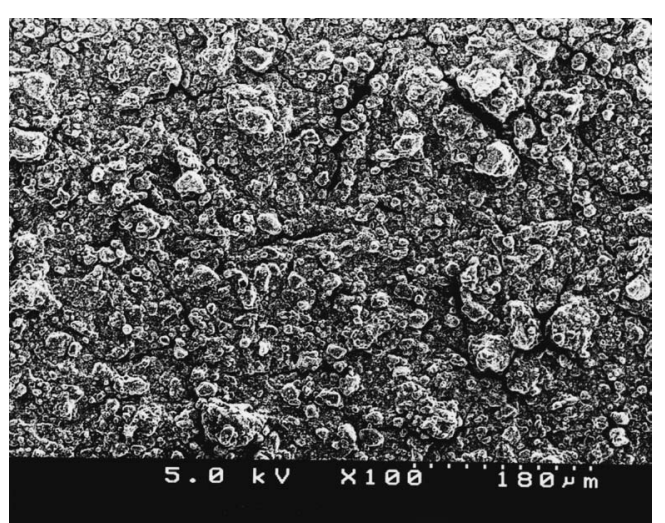

Fig. 6 Surface SEM image of the plasma-treated $\mathrm{TiO}_{2}$ film.

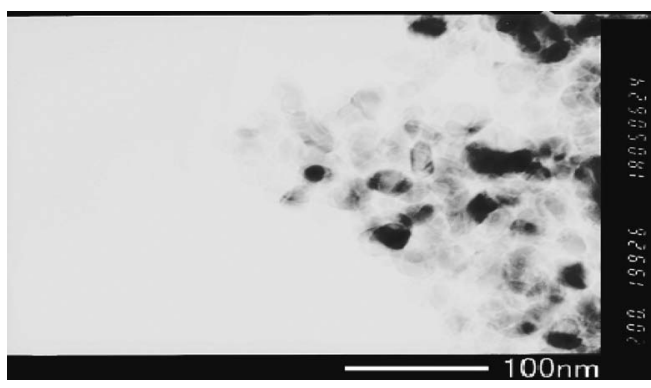

Fig. 7 TEM image of the plasma-treated $\mathrm{TiO}_{2}$ film.

by sputter deposition followed by surface treatment. Closer look to the TEM images indicates that the size of $\mathrm{TiO}_{2}$ particles is about $25 \mathrm{~nm}$. Subsequently, the $\mathrm{TiO}_{2}$ films made on these experimental bases exhibited the BET (Brunauer, Emmett, and Teller) specific surface area of $95 \mathrm{~m}^{2} / \mathrm{g}$ and the pore volume of $0.3 \mathrm{~cm}^{2} / \mathrm{g}$.

XPS is useful in elucidating surface chemistry, which may be responsible for electrochemical characteristics of the $\mathrm{TiO}_{2}$ film. Spectra of O 1 s orbital and Ti $2 \mathrm{p}$ orbital before and after plasma treatment are respectively shown in Fig. 8(a) and (b). From XPS spectra, significant increase in oxygen and titanium on the $\mathrm{TiO}_{2}$ surfaces was detected. The chemical shift of both $\mathrm{O} 1 \mathrm{~s}$ orbital and Ti 2 p orbital was carried out in high binding 

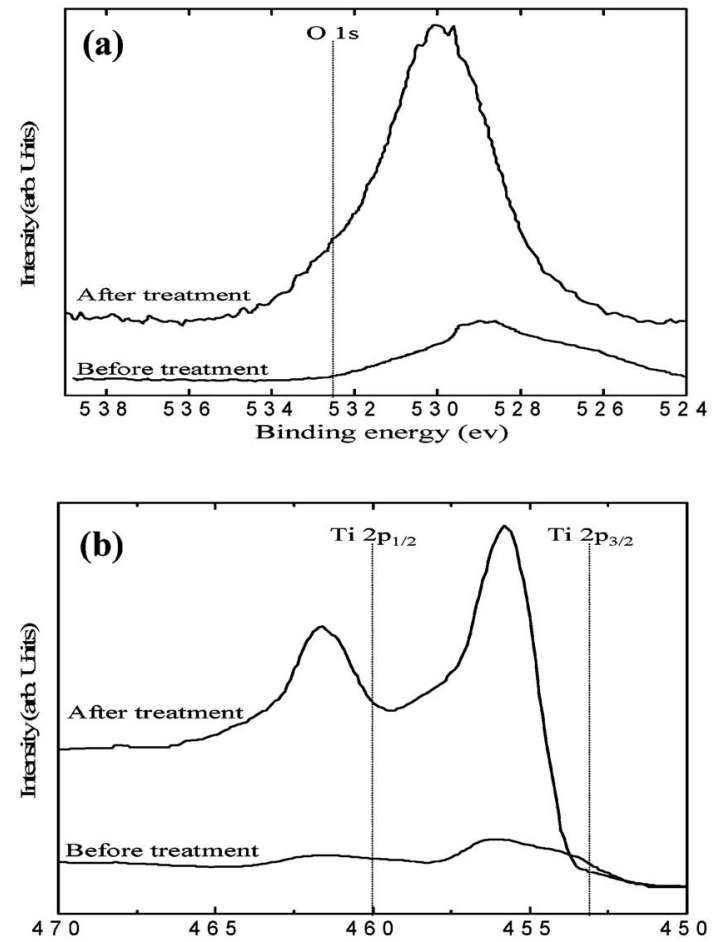

Fig. 8 XPS spectra of $\mathrm{O} 1 \mathrm{~s}$ and $\mathrm{Ti} 2 \mathrm{p}$ on the $\mathrm{TiO}_{2}$ film surface before and after plasma treatment. (a) O 1s spectra (b) Ti $2 p$ spectra

energy side. XPS measurements clearly indicate a change in chemical composition on the plasma-treated $\mathrm{TiO}_{2}$ film. The results demonstrate that a significant surface oxygen enhancement occurred by removal of the surface impurities. Oxidative treatments incorporate more oxygen onto the surface, and the electrochemical characteristics correlates well with the oxygen addition.

The photoelectric efficiency was calculated with respect to the solar spectrum through a calibration of the Light Drive 1000 lamp with direct sunlight. The overall efficiency $\eta$ of a photovoltaic cell can be calculated from the expression.

$$
\eta=\frac{J_{\mathrm{sc}} V_{\mathrm{oc}} F F}{P_{\mathrm{S}}}
$$

Where $J_{\mathrm{sc}}$ is the integral photocurrent density (current obtained at the short-circuit conditions, divided by the area of the cell), $V_{\text {oc }}$ is the open-circuit voltage, $\mathrm{FF}$ is the fill factor (related to the series resistance for a practical solar cell), and $P_{\mathrm{s}}$ is the intensity of the incident light. Figure 9 illustrates the current-voltage-power characteristics of the DSCs made of this $\mathrm{TiO}_{2}$ material.

Figure 10 shows a current per a unit area according to the DSCs with different width. The graph decreases overall as the increase of the width of DSCs. The current density is the highest at $1 \mathrm{~mm}$ of the width of DSC and falls sharply at $3 \mathrm{~mm}$. And then it decreases gradually as increasing the width of DSC to $5,7,10,15$, and $20 \mathrm{~mm}$.

In the DSC, light is absorbed in a single layer of dye molecules. After light excitation, the dye molecule injects an electron into the $\mathrm{TiO}_{2}$ film. The electron is transported to conductive glass through the $\mathrm{TiO}_{2}$ surface. It is

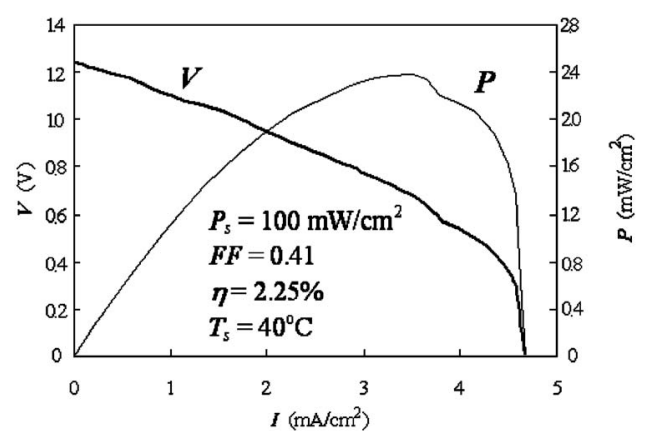

Fig. 9 I-V-P characteristics of a DSC based on $\mathrm{TiO}_{2}$ film.

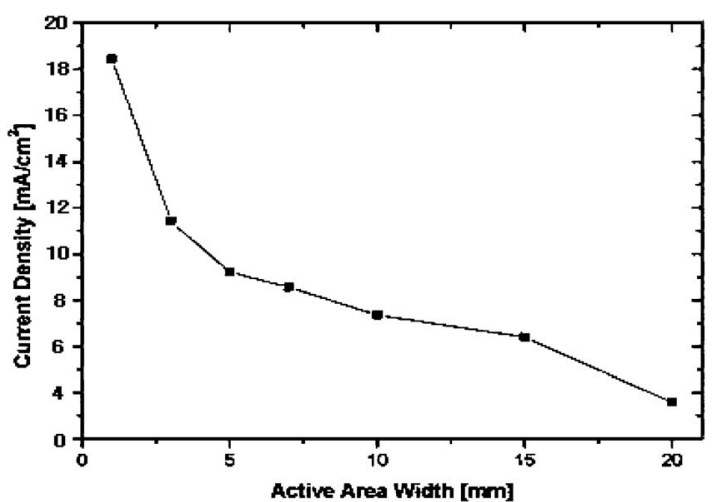

Fig. 10 The active area width vs current density of dye-sensitized solar cell samples.

collected and transferred through a load. The positive charge, formed in the light induced charge separation, is transferred from the dye to an electron donor mediator present in the cell electrolyte. Opposite of the $\mathrm{TiO}_{2}$ film is the counter electrode, where the mediator is reduced into its original state by the electron collected at $\mathrm{TiO}_{2}$ side of the cell.

There are two main factors correlated with the efficiency of the DSCs. One is the electric field generated by the incident light. The other is the transport of photon injected electrons to the collector (FTO) occurs by diffusion. But, the dominant factor which contributes to the high IPCE (incident photon-current efficiency) in DSC is generally attributed to the transport of electron diffusion. Therefore, shallow traps contribute significantly to electron transport. A diffusion phenomenon in the cell has a bad effect on IPCE of the cell because one makes internal electron transfer in a different direction. The internal electron of narrow cell has short transfer distance and is less influenced by the diffusion compared with one of the wide cell. So the narrow cell was able to be considered that the mobility of electron by electric field is relatively superior to wide cell. We regarded the movement of electron caused by the diffusion as surface resistance of the cell. In order to find out the best width of a single DSC, we have investigated the I-V characteristics of 7 different kinds of widths. An optimal cell width can be determined for given set of parameters. The current density was calculated for 7 different cell widths as shown in Fig. 2. We confirm that the increase of surface resistance 
has a bad effect on cell properties.

In this study, the sputter deposition followed by surface treatment was studied using reactive RF plasma as a method for preparing $\mathrm{TiO}_{2}$ films on FTO coated glass substrate for DSCs. The $\mathrm{TiO}_{2}$ films made on these experimental bases exhibited the BET specific surface area of $95 \mathrm{~m}^{2} / \mathrm{g}$, the pore volume of $0.3 \mathrm{~cm}^{2} / \mathrm{g}$ and the TEM particle size of $\left(25 \mathrm{~nm}\right.$. The DSCs made of this $\mathrm{TiO}_{2}$ material exhibited an energy conversion efficiency of about $2.25 \%$ at $100 \mathrm{~mW} / \mathrm{cm}^{2}$ light intensity. Consequently, we believe that the optimization between the specific surface area and photocurrent density of $\mathrm{TiO}_{2}$ films by the surface treatment was found to give rise to the improvement of energy conversion efficiency of DSCs, and the surface properties of the $\mathrm{TiO}_{2}$ significantly affect performance of DSCs.

\section{Conclusion}

We investigated electrical properties of the dye-sensitized solar cell in proportion to up sizing DSC after we had leaded to increase surface resistance by the change of width. As a result, current density of DSC was gradually decreased according to the increase of the width.

\section{Acknowledgment}

This work is supported by KOSEF (the Korea Science and Engineering Foundation) of Korea, grant No. R01-2004-000-10318-0.

\section{References}

1) A. Kay and M. Gratzel: Solar Energy Mater. Solar Cells, 44 (1996) 99.

2) B. O'Regan and M. Gr.atzel: Nature, 353 (1991) 737.

3) M. Gomez, et al.: Sol. Energy Mater. Sol. Cells, 64 (2000) 385.

4) M. Späth, P. M. Sommeling, J. A. M. van Roosmalen, et al.: Progress in photovolt. res. appl., 11 (2003) 207.

5) Michael Grätzel: Progress in photovolt. res. appl., 8 (2000) 171.

6) J. S. Kim, M. GransTrom, R. H. Friend, N. Johansson, W. R. Salaneck, R. Daik, W. J. Feast and F. Cacialli: J. Appl. Phys., 84 (1998) 6859.

7) J. S. Kim, R. H. Friend and F. Cacialli: Appl. Phys. Lett., 74 (1999) 3084.

8) Y. M. Sung, M. Otsubo, C. Honda and C. H. Park: Jpn. J. Appl. Phys., 41 (2002) 6563. 